\title{
MÉMENTO TECHNIQUe À L'USAGE DES BIOLOGISTES ET BIOCHIMISTES
}




\section{Grenoble Sciences}

Grenoble Sciences est un centre de conseil, expertise et labellisation de l'enseignement supérieur français. Il expertise les projets scientifiques des auteurs dans une démarche à plusieurs niveaux (référés anonymes, comité de lecture interactif) qui permet la labellisation des meilleurs projets après leur optimisation. Les ouvrages labellisés dans une collection de Grenoble Sciences ou portant la mention «Sélectionné par Grenoble Sciences» («Selected by Grenoble Sciences») correspondent à :

- des projets clairement définis sans contrainte de mode ou de programme,

- des qualités scientifiques et pédagogiques certifiées par le mode de sélection (les membres du comité de lecture interactif sont cités au début de l'ouvrage),

- une qualité de réalisation assurée par le centre technique de Grenoble Sciences.

\section{Directeur scientifique de Grenoble Sciences}

Jean BORnarel, Professeur émérite à l'Université Joseph FoURIER, Grenoble 1

On peut mieux connaître Grenoble Sciences en visitant le site web :

$$
\text { https://grenoble-sciences.ujf-grenoble.fr }
$$

On peut également contacter directement Grenoble Sciences :

Tél : (33) 4765146 95, e-mail : grenoble.sciences@ujf-grenoble.fr

\section{Livres et pap-ebooks}

Grenoble Sciences labellise des livres papier (en langue française et en langue anglaise) mais également des ouvrages utilisant d'autres supports. Dans ce contexte, situons le concept de pap-ebook qui se compose de deux éléments :

- un livre papier qui demeure l'objet central avec toutes les qualités que l'on connait au livre papier,

- un site web compagnon qui propose :

$>$ des éléments permettant de combler les lacunes du lecteur qui ne possèderait pas

les prérequis nécessaires à une utilisation optimale de l'ouvrage,

$>$ des exercices pour s'entraîner,

> des compléments pour approfondir un thème, trouver des liens sur internet, etc.

Le livre du pap-ebook est autosuffisant et certains lecteurs n'utiliseront pas le site web compagnon. D'autres l'utiliserons et ce, chacun à sa manière. Un livre qui fait partie d'un pap-ebook porte en première de couverture un logo caractéristique et le lecteur trouvera le site compagnon de ce livre à l'adresse internet suivante :

https://grenoble-sciences.ujf-grenoble.fr/pap-ebooks/marouf-tremblin

Grenoble Sciences bénéficie du soutien du Ministère de l'Enseignement supérieur et de la Recherche et de la Région Rhône-Alpes.

Grenoble Sciences est rattaché à l'Université Joseph FourIER de Grenoble.

ISBN 978-2-7598-0965-3

(C) EDP Sciences, 2013 


\section{MÉMento TECHNIQUe À L'USAGE DES BIOLOGISTES ET BIOCHIMISTES}

\section{Abderrazak Marouf et Gérard Tremblin}

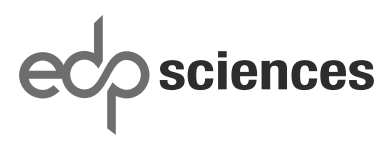

17, avenue du Hoggar

Parc d'Activité de Courtabœuf - BP 112

91944 Les Ulis Cedex A - France 